\title{
ON THE DENSITY OF SOME SEQUENCES OF INTEGERS
}

\section{P. ERDÖS}

Let $a_{1}<a_{2}<\cdots$ be any sequence of integers such that no one divides any other, and let $b_{1}<b_{2}<\cdots$ be the sequence composed of those integers which are divisible by at least one $a$. It was once conjectured that the sequence of $b$ 's necessarily possesses a density. Besicovitch ${ }^{1}$ showed that this is not the case. Later Davenport and $\mathrm{I}^{2}$ showed that the sequence of $b$ 's always has a logarithmic density, in other words that $\lim _{n \rightarrow \infty}(1 / \log n) \sum_{b_{i} \leqq n} 1 / b_{i}$ exists, and that this logarithmic density is also the lower density of the $b$ 's.

It is very easy to see that if $\sum 1 / a_{i}$ converges, then the sequence of $b$ 's possesses a density. Also it is easy to see that if every pair of $a$ 's is relatively prime, the density of the $b^{\prime}$ 's equals $\mathrm{II}\left(1-1 / a_{i}\right)$, that is, is 0 if and only if $\sum 1 / a_{i}$ diverges. In the present paper I investigate what weaker conditions will insure that the $b$ 's have a density. Let $f(n)$ denote the number of $a$ 's not exceeding $n$. I prove that if $f(n)<c n / \log n$, where $c$ is a constant, then the $b$ 's have a density. This result is best possible, since we show that if $\psi(n)$ is any function which tends to infinity with $n$, then there exists a sequence $\mathrm{a}_{n}$ with $f(n)<n \cdot \psi(n) / \log n$, for which the density of the $b$ 's does not exist. The former result will be obtained as a consequence of a slightly more precise theorem. Let $\phi\left(n ; x ; y_{1}, y_{2}, \cdots, y_{n}\right)$ denote generally the number of integers not exceeding $n$ which are divisible by $x$ but not divisible by $y_{1}, \cdots, y_{n}$. Then a necessary and sufficient condition for the $b$ 's to have a density is that

$$
\lim _{\epsilon \rightarrow 0} \limsup _{n \rightarrow \infty} \frac{1}{n} \sum_{n^{1-\epsilon<a_{i} \leqq n}} \phi\left(n ; a_{i} ; a_{1}, a_{2}, \cdots, a_{i-1}\right)=0 .
$$

The condition (1) is certainly satisfied if $f(n)<c n / \log n$, since

$$
\begin{aligned}
\frac{1}{n} \sum_{n^{1-\epsilon<a_{i} \leqq n}} \phi\left(n ; a_{i} ; a_{1} \cdots a_{i-1}\right) & <\frac{1}{n} \sum_{n^{1-\epsilon<a_{i} \leqq n}}\left[\frac{n}{a_{i}}\right] \\
& <\sum_{n^{1-\epsilon}<m \log m<n} \frac{c^{\prime}}{m \log m}=O(\epsilon)+O\left(\frac{1}{n}\right) .
\end{aligned}
$$

Received by the editors April 28, 1947, and, in revised form, September 5, 1947.

1 Math. Ann. vol. 110 (1934-1935) pp. 336-341.

2 Acta Arithmetica vol. 2. 
As an application of the condition (1) we shall prove that the set of all integers $m$ which have two divisors $d_{1}, d_{2}$ satisfying $d_{1}<d_{2} \leqq 2 d_{1}$ exists. I have long conjectured that this density exists, and has value 1 , but have still not been able to prove the latter statement.

At the end of the paper I state some unsolved problems connected with the density of a sequence of positive integers.

Theorem 1. Let $\psi(n) \rightarrow \infty$ as $n \rightarrow \infty$. Then there exists a sequence $a_{1}<a_{2}<\cdots$ of positive integers such that no one of them divides any other, with $f(n)<n \psi(n) / \log n$, and such that the sequence of b's does not have a density.

Proof. We observe first that the condition that one $a$ does not divide another is inessential here, since we can always select a subsequence having this property, such that every $a$ is divisible by at least one $a$ of the subsequence. The condition on $f(n)$ will remain valid, and the sequence of $b$ 's will not be affected.

Let $\epsilon_{1}, \epsilon_{2}, \cdots$ be a decreasing sequence of positive numbers, tending to 0 sufficiently rapidly, and let $n_{r}=n_{r}\left(\epsilon_{r}\right)$ be a positive integer which we shall suppose later to tend to infinity sufficiently rapidly. We suppose that $n_{r}^{1-\epsilon_{r}}>n_{r-1}$ for all $r$. We define the $a$ 's to consist of all integers in the interval $\left(n_{r}^{1-\epsilon_{r}}, n_{r}\right)$ which have all their prime factors greater than $n_{r}^{2}$, for $r=1,2, \cdots$.

We have first to estimate $f(m)$, the number of $a$ 's not exceeding $m$. Let $r$ be the largest suffix for which $n_{r}^{1-\epsilon_{r}} \leqq m$. If $m \geqq n_{r}^{2}$, then clearly

$$
f(m)<n_{r} \leqq m^{1 / 2}<\frac{m}{\log m} .
$$

Suppose, then, that $m<n_{r}^{2}$. We have

$$
f(m)<n_{r-1}+M_{\epsilon}(m),
$$

where $M_{\epsilon}(m)$ denotes the number of integers not exceeding $m$ which have all their prime factors greater than $m^{e_{r}^{2} / 2}$. By Brun's ${ }^{3}$ method we obtain

$$
M_{\epsilon}(m)<c_{1} m \sum_{p \leqq m \epsilon_{r}^{2} / 2}\left(1-p^{-1}\right)<c_{2} \frac{m}{\epsilon_{r}^{2} \log m},
$$

where $c_{1}, c_{2}$, denote positive absolute constants. Hence

$$
f(m)<n_{r-1}+c_{2} \frac{m}{\epsilon_{r}^{2} \log m}<\frac{n \psi(m)}{\log m}
$$

${ }^{3}$ P. Erdös and M. Kac, Amer. J. Math. vol. 62 (1940) pp. 738-742. 
provided $n_{r}\left(\epsilon_{r}\right)$ is sufficiently large. It will suffice if

$$
\frac{c_{2}}{\epsilon_{r}^{2}}<\frac{1}{2} \psi\left(n_{r}^{1-\epsilon_{r}}\right) .
$$

We have now to prove that the sequence of $b$ 's (the multiples of the $a$ 's) have no density. Denote by $A(\epsilon, n)$ the density of the sequence of all integers which have at least one divisor in the interval $\left(n^{1-\epsilon}, n\right)$. In a previous paper ${ }^{4}$ proved that $A(\epsilon, n) \rightarrow 0$ if $\epsilon \rightarrow 0$ and $n \rightarrow \infty$ independently. Thus if $\epsilon \rightarrow 0$ and $n \rightarrow \infty$ sufficiently fast, we have

$$
\sum_{r=1}^{\infty} A\left(\epsilon_{r}, n_{r}\right)<\frac{1}{2} .
$$

Denote the number of $b$ 's not exceeding $m$ by $B(m)$. It follows from (2) that if $n_{r} \rightarrow \infty$ sufficiently rapidly, and $m=n_{r}^{1-\epsilon_{r}}$, then

$$
B(m)<m / 2 \text {. }
$$

This proves that the lower density of the $b$ 's is at most $1 / 2$.

Next we show that the upper density of the $b$ 's is 1 , and this will complete the proof of Theorem 1. It suffices to prove that

$$
n_{r}-B\left(n_{r}\right)=o\left(n_{r}\right),
$$

in other words that the number of integers up to $n_{r}$ which are not divisible by any $a$ is $o\left(n_{r}\right)$. Consider any integer $t$ satisfying $n_{r}^{1-\epsilon_{r} / 2}<t$ $\leqq n_{r}$, and define

$$
\left(g_{\epsilon_{r}}(t)\right)=g_{r}(t)=\prod_{p}^{\prime} p^{\alpha},
$$

where the dash indicates that the product is extended over all primes $p$ with $p \leqq n_{r}^{e_{r}^{2}}$, and $p^{\alpha}$ is the exact power of $p$ dividing $t$.

If $g_{r}(t)<n^{\epsilon_{r} / 2}$, then $t$ is divisible by an $a$, since $t / g_{r}(t)>n^{1-\epsilon_{r}}$ and $t / g_{r}(t)$ has all its prime factors greater than $n_{r}^{\epsilon_{r}^{2}}$, and so is an $a$. Hence

$$
n_{r}-B\left(n_{r}\right)<n_{r}^{1-\epsilon_{r} / 2}+C\left(n_{r}\right),
$$

where $C\left(n_{r}\right)$ denotes the number of integers $t \leqq n_{r}$ for which $g_{r}(t)$ $\geqq n_{r}^{\epsilon_{r} / 2}$. We recall that the exact power of a prime $p$ dividing $N$ ! is

$$
\sum_{\nu=1}^{\infty}\left[\frac{N}{p^{\nu}}\right]<\sum_{\nu=1}^{\infty} \frac{N}{p^{\nu}}=\frac{N}{p-1} .
$$

Hence

${ }^{4}$ J. London Math. Soc. vol. 11 (1936) pp. 92-96. 


$$
\begin{aligned}
& \prod_{t=1}^{n_{r}} g_{r}(t) \leqq \prod_{p \leqq n \epsilon_{r}^{2}} p^{n_{r} / p-1}=\exp \left(n_{r} \sum_{p \leqq n \epsilon_{r}^{2}} \frac{\log p}{p-1}\right. \\
& <\exp \left(c_{3} \epsilon_{r}^{2} n_{r} \log n_{r}\right)=n_{r}^{c_{3} \epsilon_{r}^{2} n_{r}} .
\end{aligned}
$$

Hence $\left(n_{r}^{\epsilon_{r} / 2}\right)^{C\left(n_{r}\right)}<n_{r}^{c_{3} \epsilon_{r}^{2} n_{r}}$, whence

$$
C\left(n_{r}\right)<2 c_{3} \epsilon_{r} n_{r}
$$

Substituted in (5), this proves (4), provided that $n_{r}^{\epsilon_{r}} \rightarrow \infty$, which we may suppose to be the case. This completes the proof of Theorem 1 .

THEOREM 2. A necessary and sufficient condition that the b's shall have a density is that (1) shall hold.

Proof. The necessity is easily deduced from an old result. Davenport and $\mathrm{I}^{2}$ proved that the logarithmic density of the $b$ 's exists and has the value

$$
\lim _{i \rightarrow \infty} \lim _{n \rightarrow \infty} \frac{1}{n} \sum_{j \leqq i} \phi\left(n ; a_{j} ; a_{1}, \cdots, a_{j-1}\right) .
$$

Thus if the density of the b's exists, we obtain

$$
\lim _{i \rightarrow \infty} \lim _{n \rightarrow \infty} \frac{1}{n} \sum_{j>i} \phi\left(n ; a_{j} ; a_{1}, \cdots, a_{j-1}\right)=0 .
$$

This proves the necessity of (1).

The proof of the sufficiency is much more difficult. We have

$$
B(n)=\sum_{a_{i} \leqq n} \phi\left(n ; a_{i} ; a_{1}, \cdots, a_{i-1}\right)=\sum_{1}+\sum_{2}+\sum_{3},
$$

where $\sum_{1}$ is extended over $a_{i} \leqq A, \sum_{2}$ over $A<a_{i} \leqq n^{1-\epsilon}, \sum_{3}$ over $n^{1-\epsilon}<a_{i} \leqq n$. Here $A=A(n)$ will be chosen later to tend to infinity with $n$. By the hypothesis (1) we have

$$
\lim _{\epsilon \rightarrow 0} \limsup _{n \rightarrow \infty} \frac{1}{n} \sum_{3}=0 .
$$

It follows from the earlier work ${ }^{2}$ that if $A=A(n)$ tends to infinity sufficiently slowly, then $(1 / n) \sum_{1}$ has a limit, this limit being the logarithmic density of the $b$ 's, and also

$$
\lim _{j \rightarrow \infty}\left(\sum_{i \leqq j} \frac{1}{a_{i}}-\sum_{i_{1}<i_{2} \leqq j} \frac{1}{\left[a_{i_{1}}, a_{i_{2}}\right]}+\cdots\right) .
$$

Thus the proof of Theorem 2 will be complete if we are able to prove that 


$$
\frac{1}{n} \sum_{2}=\lim _{\epsilon \rightarrow 0} \limsup _{n \rightarrow \infty} \frac{1}{n} \sum_{A<a_{i} \leqq n^{1-\epsilon}} \phi\left(n ; a_{i} ; a_{1}, \cdots, a_{i-1}\right)=0 .
$$

We have

$$
\phi\left(n ; a_{i} ; a_{1}, \cdots, a_{i-1}\right)=\phi\left(\frac{n}{a_{i}}, 1 ; d_{1}^{(i)} \cdots\right)
$$

where

$$
d_{j}^{(i)}=\frac{a_{j}}{\left(a_{i}, a_{j}\right)}
$$

We shall prove that

$$
\lim _{\epsilon \rightarrow 0} \limsup _{n \rightarrow \infty} \frac{1}{n} \sum_{A<a_{i} \leqq n^{1-\epsilon}} \phi^{\prime}\left(\frac{n}{a_{i}} ; 1 ; d_{1}^{(i)} \ldots\right)=0
$$

where the dash indicates that we retain only those $d_{j}^{(i)}$ which satisfy $d_{j}^{(i)}<n^{\epsilon^{2}}$. Clearly (8) follows from (9). (Since $n^{\epsilon^{2}} \rightarrow \infty$, not all the $d_{j}^{(i)}$ are greater than or equal to $n^{\epsilon^{2}}$.)

We define $g_{\epsilon}(t)$ as before, with $n$ in place of $n_{r}$ and $\epsilon$ in place of $\epsilon_{r}$. It follows from (5) and (6) that it will suffice to prove that

$$
\lim _{\epsilon \rightarrow 0} \limsup _{n \rightarrow \infty} \frac{1}{n} \sum_{A<t_{i} \leqq n^{1-\epsilon}} \phi^{\prime \prime}\left(\frac{n}{a_{i}} ; 1 ; d_{1}^{(i)} \ldots\right)=0,
$$

where $\phi^{\prime \prime}\left(n / a_{i} ; 1 ; d_{1}^{(t)} \cdots\right)$ denotes the number of integers $m$ satisfying

$$
\text { (11) } \quad m \leqq \frac{n}{a_{i}} ; \quad m \neq 0\left(\bmod d_{j}^{(i)}\right), \quad d_{j}^{(i)}<n^{\epsilon^{2}} ; \quad g_{\epsilon}(m)<n^{\epsilon / 2} .
$$

Consider the integers satisfying (11). They are of the form $u \cdot v$ where $u<n^{\epsilon / 2}$ and all prime factors of $u$ are less than $n^{\epsilon^{2}}, u \neq 0\left(\bmod d_{j}^{(\gamma)}\right)$ for $d_{j}^{(1)}<n^{\epsilon^{2}}$, and all prime factors of $v$ are greater than $n^{\epsilon^{2}}$. We obtain by Brun's method $^{3}$ that the number of integers $m \leqq n / a_{i}$ with fixed $u$ does not exceed $\left(n / u \cdot a_{i}>n^{\epsilon / 2}\right)$

$$
c_{4} \frac{n}{a_{i} u} \prod_{p<n \epsilon^{2}}\left(1-p^{-1}\right) .
$$

Thus the number $N_{i}$ of integers satisfying (11) does not exceed

$$
c_{4} \frac{n}{a_{i}} \sum{ }^{\prime} \frac{1}{u} \prod_{p<n \epsilon^{2}}\left(1-p^{-1}\right) \geqq \phi^{\prime \prime}\left(\frac{n}{a_{i}} ; 1 ; d_{1}^{(i)} \cdots\right),
$$


where the dash indicates that the summation is extended over the $u<n^{\epsilon / 2}, u \neq 0\left(\bmod d_{j}^{(i)}\right), d_{j}^{(i)}<n^{\epsilon^{2}}$ and all prime factors of $u$ are less than $n^{\epsilon^{2}}$.

We have to estimate $\sum N_{i}$. Put

$$
\lim _{m \rightarrow \infty} \frac{1}{m} \phi\left(\frac{m}{a_{i}} ; 1 ; d_{1}^{(i)} \cdots\right)=t_{i},
$$

where in (14) all the $d_{j}^{(i)}$ are considered. (It follows from the definition of the $d_{j}^{(i)}$ that they are all less than $n$. Thus the limit (14) exists.) It follows from our earlier work ${ }^{2}$ that

$$
\sum_{a_{i}>A} t_{i}=o(1)
$$

Next we estimate $t_{i}^{\prime}$ where

$$
t_{i}^{\prime}=\lim _{m \rightarrow \infty} \frac{1}{m} \phi\left(\frac{m}{a_{i}} ; 1 ; d_{j}^{(i)}\right), \quad d_{j}^{(i)}<n^{\epsilon^{2}} .
$$

Here we use the following result of Behrend ${ }^{5}$

$$
\begin{aligned}
\lim _{n \rightarrow \infty} \frac{1}{n} \phi\left(n ; 1 ; a_{1}, \cdots, a_{i}, b_{1}, \cdots, b_{i}\right) \\
\quad \geqq \lim _{n \rightarrow \infty} \frac{1}{n^{2}} \phi\left(n ; 1 ; a_{1}, \cdots, a_{i}\right) \cdot \phi\left(n ; 1 ; b_{1}, \cdots, b_{i}\right) \cdots .
\end{aligned}
$$

Thus clearly

$$
t_{i}^{\prime} \leqq t_{i}\left(\lim _{m \rightarrow \infty} \frac{1}{m} \phi\left(m ; 1 ; x_{i^{r}}\right)^{-1}=t_{i} / t_{i}^{\prime \prime},\right.
$$

where $x_{i}$ runs through the integers from $n^{\epsilon^{2}}$ to $n$. It follows from the Sieve of Eratosthenes that the density of integers with $g_{e}(m)=k$ equals

$$
\frac{1}{k} \prod_{p<n \epsilon^{2}}\left(1-p^{-1}\right)
$$

Thus clearly

$$
t_{i}^{\prime \prime} \geqq \sum_{k<n^{\epsilon^{2}}} \frac{1}{k} \prod_{p \geqq n}\left(1-p^{-1}\right)>c_{5} \epsilon^{2}
$$

or

$$
t_{i}^{\prime} \leqq t_{i} / c_{5} \epsilon^{2}
$$

${ }^{5}$ Bull. Amer. Math. Soc. vol. 54 (1948) pp. 681-684. 
Thus from (15) and (17),

$$
\sum_{a_{i}>A} t_{i}=o(1)
$$

We have by the Sieve of Eratosthenes

$$
t_{i}^{\prime}=\frac{1}{a_{i}} \sum^{\prime} \frac{1}{x} \prod_{p<n \epsilon^{2}}\left(1-p^{-1}\right)
$$

where the dash indicates that $x \neq 0\left(\bmod d_{j}^{(i)}\right) d_{j}^{(i)}<n^{\epsilon^{2}}$ and all prime factors of $x$ are less than $n^{\epsilon^{2}}$. Comparing (13) and (19) we obtain

$$
N_{i}<c_{4} t_{i}^{\prime} n \text {. }
$$

Thus finally from (10) and (18) we obtain $\sum_{a_{i}>A} N_{i}=o(n)$ which proves (10) and completes the proof of Theorem 2.

THEOREM 3. The density of integers having two divisors $d_{1}$ and $d_{2}$ with $d_{1}<d_{2}<2 d_{1}$ exists.

Proof. Define a sequence $a_{1}, a_{2}, \cdots$ of integers as follows: An integer $m$ is an $a$ if $m$ has two divisors $d_{1}$ and $d_{2}$ with $d_{1}<d_{2}<2 d_{1}$, but no divisor of $m$ has this property. To prove Theorem 3 it will be sufficient to show that the multiples of the $a$ 's have a density. Thus by Theorem 2 we only have to show that (1) is satisfied. We shall only sketch the proof.

Clearly the $a$ 's are of the form $x y$, where $x<y<2 x$. Thus it will be sufficient to show that the number of integers $m \leqq n$ having a divisor in the interval $\left(n^{1 / 2-\epsilon}, n^{1 / 2}\right)$ is less than $\eta n$ where $\eta \rightarrow 0$ as $\epsilon \rightarrow 0$. But I proved that the density $c_{\epsilon, t}$ of integers having a divisor in $\left(t, t^{1+\epsilon}\right)$ satisfies

$$
\lim _{\epsilon \rightarrow 0} \lim _{t \rightarrow \infty} c_{\epsilon, t}=0
$$

A similar argument will prove the above result, and so complete the proof of Theorem 3.

It can be shown that the density of integers having two divisors $d_{1}$ and $d_{2}$ with $d_{1}<d_{2} \leqq 2 d_{1}$ and either $d_{1}$ or $d_{2}$ a prime exists and is less than 1. This result is not quite trivial, since if we denote by $a_{1}<a_{2}<\cdots$ the sequence of those integers having this property and such that no divisor of any $a$ has this property, then $\sum 1 / a_{i}$ diverges.

We now state a few unsolved problems.

I. Besicovitch ${ }^{1}$ constructed a sequence $a_{1}<a_{2}<\cdots$ of integers such that no $a$ divides any other, and the upper density of the $a$ 's 
is positive. A result of Behrend ${ }^{6}$ states that

$$
\lim \frac{1}{\log n} \sum_{a_{i} \leqq n} \frac{1}{a_{i}}=0
$$

and $I^{7}$ proved that

$$
\sum \frac{1}{a_{i} \log a_{i}}<A
$$

where $A$ is an absolute constant. It follows from the last two results that the lower density of the $a$ 's must be 0 . In fact Davenport and $\mathrm{I}^{2}$ proved the following stronger result: Let $d_{1}<d_{2}<\cdots$ be a sequence of integers of positive logarithmic density, then there exists an infinite subsequence $d_{i_{1}}<d_{i_{2}}<\cdots$ such that $d_{i_{j}} \mid d_{i_{j+i}}$. Let now $f_{1}<f_{2}<\cdots$ be a sequence of positive lower density. Can we always find two numbers $f_{i}$ and $f_{j}$ with $-f_{i} \nmid f_{j}$ and so that $\left[f_{i} \mid f_{j}\right]$ also belongs to the sequence? This would follow if the answer to the following purely combinatorial conjecture is in the affirmative: Let $c$ be any constant and $n$ large enough. Consider $c 2^{n}$ subsets of $n$ elements. Then there exist three of these subsets $B_{1}, B_{2}, B_{3}$ such that $B_{3}$ is the union of $B_{1}$ and $B_{2}$.

II. Let $a_{1}<a_{2}<\cdots$ be a sequence of real numbers such that for all integers $k, i, j$ we have $\left|k a_{i}-a_{j}\right| \geqq 1$. Is it then true that $\sum 1 / a_{i} \log a_{i}$ converges and that $\lim (1 / \log n) \sum_{a_{i<} n} 1 / a_{i}=0$ ? If the $a$ 's are all integers the condition $\left|k a_{j}-a_{s}\right| \geqq 1$ means that no $a$ divides any other, and in this case our conjectures are proved by (21) and (22).

III. Let $a_{1}<a_{2}<\cdots \leqq n$ be any sequence of integers such that no one divides any other, and let $m>n$. Denote by $B(m)$ the number of $b$ 's not exceeding $m$. Is it true that

$$
\frac{B(m)}{m}>\frac{1}{2} \frac{B(n)}{n} \text { ? }
$$

It is easy to see that the constant 2 can not be replaced by any smaller one. (Let the $a$ 's consist of $a_{1}$ and $n=a_{1}, m=2 a_{1}-1$.)

I was unable to prove or disprove any of these results.

Syracuse University

${ }^{6} \mathrm{~J}$. London Math. Soc. vol. 10 (1935) pp. 42-44.

7 Ibid. vol. 10 (1935) pp. 126-128. 